\title{
A NEW MICROPHOTOMETER FOR PHOTOGRAPHIC DENSITIES
}

\author{
By W. F. Meggers and Paul D. Foote
}

\section{CONTENTS}

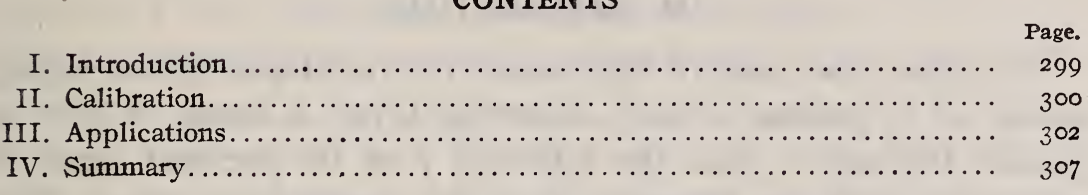

\section{INTRODUCTION}

Problems involving the necessity of measuring the photographic density of a minute portion of a photographic plate have called into existence several types of microphotometers such as the well-known Hartmann ${ }^{1}$ instrument, the Koch ${ }^{2}$ registering microphotometer, and the new microphotometer recently described by Fabry and Buisson. ${ }^{3}$

In the course of some recent experiments on low voltage arc spectra of cæsium, the writers required the use of a microphotometer to measure photographic densities of spectral lines. None of the above-mentioned instruments being at hand, the instrument which was employed by the writers over three years ago in some photographic density work, by the addition of a few slight changes, proved so satisfactory, accurate, and efficient a microphotometer as to merit

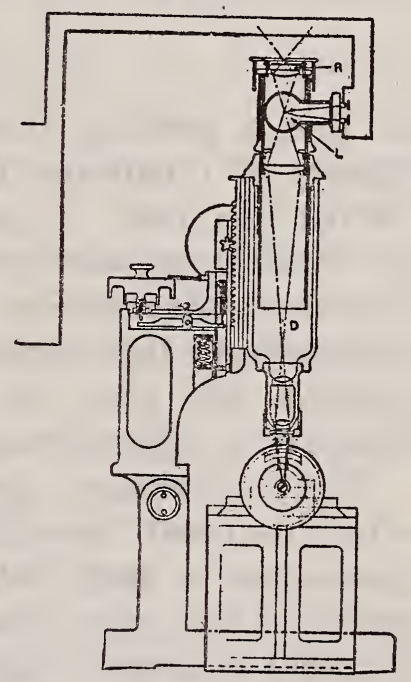

FIG. I.-Microphotometer serious consideration as a piece of laboratory apparatus. The instrument, Fig. I, is essentially the micropyrometer described by Burgess ${ }^{4}$ except that a microscope of higher power was employed. The photographic plate was mounted, just below the objective of the microscope, on a horizontal bed movable with a

1 Zeitschr. f. Inst. 19, p. $97 ;$ 1899.

Ann. d. Phys. (4), 39, p. 705; I9r2. $182091^{\circ}-20$
3 J. de Phys. (5), 9, p. 37; r9r9.

B. S. Bulletin, 9, p. 475; I913 (Scientific Paper No. 198). 
graduated screw, and was illuminated beneath by an intense beam of light from a tungsten ribbon gas-filled lamp. Light transmitted by a small portion of the photographic plate, the image of which was adjacent to that of the tip of the photometer lamp, was matched with equal filament brightness by adjusting the current through the lamp. The ammeter readings are readily translated into measurements of photographic density.

\section{CALIBRATION}

To define the state of blackness of a developed photographic plate, or in general of any absorbing layer, a beam of light is passed through it, and the intensity $I$ of the incident beam is compared with the intensity $T$ of the transmitted beam. The ratio $O=I / T$ is called the opacity and the value of this ratio is $\mathrm{r}$ for a perfectly transparent plate. It is more convenient, however, to use the logarithm (taken to the base ro) of this quantity and call it density $D$. Then:

$$
D=\log O=\log I / T
$$

so that a perfectly transparent plate has zero density, and a density of $\mathrm{r}$ indicates that $\mathrm{r} / \mathrm{ro}$ of the incident light is transmitted by a plate. In general, slightly different values of density are found from measurements with different colored lights. It is, therefore, advantageous to make density measurements with homogeneous light rather than with white light, since the phenomena then obey the above simple law, and the difficulties inherent in heterochromatic photometry are evaded.

These advantages are possessed by the microphotometer in which the usual "monochromatic" red-glass screen used in optical pyrometers is employed. Of course, a suitable monochromatic screen of any other color could be used in the same way. The densities measured with red light will be the same as for any other color if the photographic plate is nonselective.

There are several methods of calibration and use of this type of photometer of which the following appeared the most satisfactory. The instrument with a red-glass ocular was calibrated as a pyrometer by sighting upon a small black body, the temperature of which was measured by an optical pyrometer, thus obtaining the relation between the temperature of the source and the current through the photometer lamp sufficient for a match. If the red-glass screen is sufficiently monochromatic, we may neglect the variation 
of the "effective wave length" of transmission with the temperature of the source. ${ }^{5}$ The particular glass employed had an effective wave length of approximately $\lambda=0.65 \mu$.

In use the illuminating source is maintained at any constant brightness sufficiently high to permit observation on lines of the highest density. The measurements of the current required to match against any line and against the clear film in the neighborhood of this line, are made and are converted into "temperatures" by use of the calibration plot of the instrument. If $\theta_{1}$ is the absolute temperature observed for the line and $\theta_{2}$ the correspond-

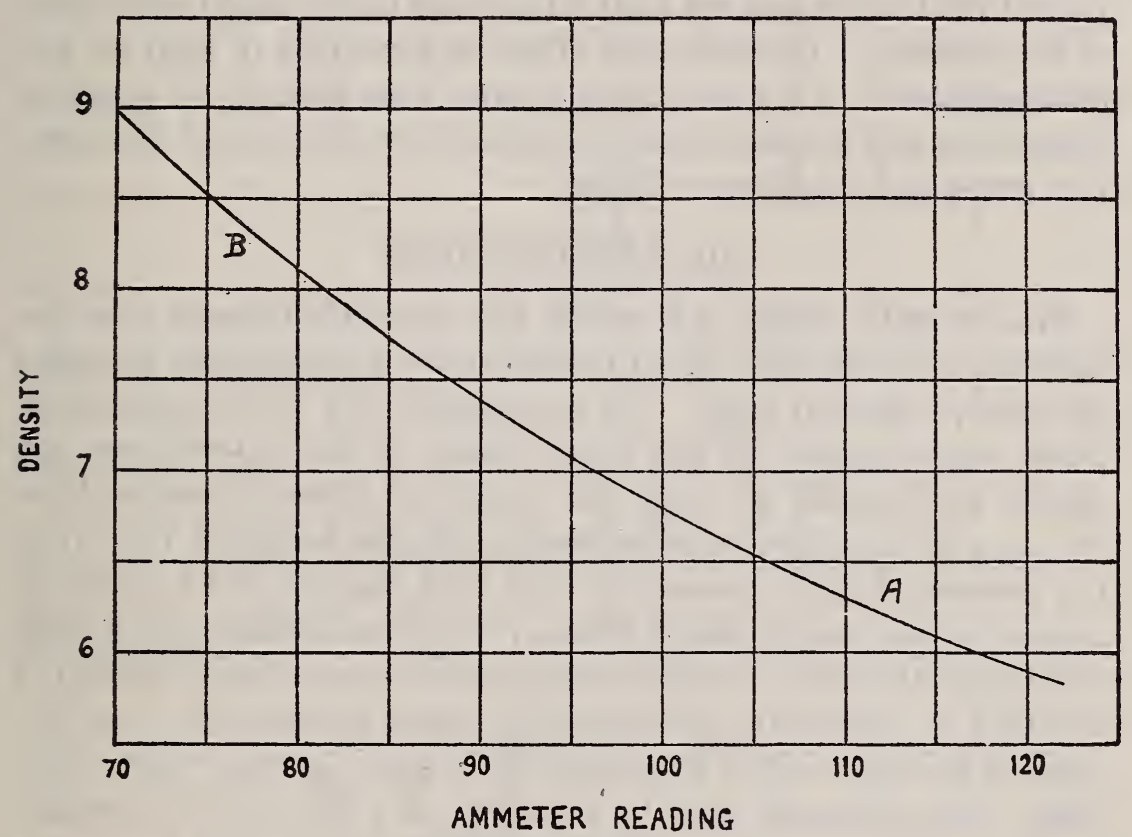

FIG. 2.-Calibration curve of microphotometer

ing temperature for the clear film (fog) the true density $D$ of the line, fog correction being considered, follows at once from Wien's law:

$$
D=\frac{c_{2} \log e}{\lambda}\left(\frac{\mathrm{I}}{\theta_{1}}-\frac{\mathrm{I}}{\theta_{2}}\right)=9590\left(\frac{\mathrm{I}}{\theta_{1}}-\frac{\mathrm{I}}{\theta_{2}}\right)
$$

For convenience of use, instead of plotting current through the lamp versus temperature, we plot current versus $9590 / \theta$, obtaining for the particular instrument employed, the curve shown in Fig. 2. Thus, suppose the current $i_{\mathrm{A}}$ is observed for any fog reading on

6 B. S. Bulletin, 12, p. 483; 1915 (Scientific Paper No. 260). 
clear film and $i_{\mathrm{B}}$ when sighted upon the spectral line. The true density of the line is given by the difference in ordinates of the points $A$ and $B$.

Another method of calibration, in some respects more satisfactory, is to sight upon the illuminating source and vary the intensity by means of sectored disks. Still another method is to use screens which have been measured by other photometers. Screens calibrated for white light may be employed when plates similarly treated are measured, thus eliminating the slight difference in density arising from measurements in the usually employed white light and red light on account of any slight selectivity of the gelatine. However, the effect of scattering of light by the photographic film is many times greater than that of the selective absorption and requires special attention to the type of illumination employed, as discussed later.

\section{APPLICATIONS}

The primary object for which this microphotometer was assembled and calibrated was to measure the photographic densities of narrow spectral lines. To accomplish this the photographic plate was mounted in the object plane of the microscope and moved horizontally to bring the images of different spectral lines or parts of the plate in coincidence with the image of the tip of the pyrometer lamp filament. This shift was effected by turning a screw which was provided with a revolution counter, and a head divided so that one division corresponds to $5 \mu$ in linear motion of the bed on which the photographic plate is mounted. An important feature of this arrangement is that the tip of the pyrometer lamp filament may be regarded as a filar in the eyepiece and the relative positions or wave lengths of spectral lines on a plate are measured at the same time that their photographic densities are measured. The measurement of densities has been explained above and it only remains to give an example of the simultaneous double use of this instrument as a photo measuring micrometer and a microphotometer. Table I gives the results obtained from a one-minute exposure of a dicyanin stained plate to a 6-volt cæsium arc spectrum formed by a small prism spectrograph.

The first three columns of Table I illustrate the use of the microphotometer for wave-length measurements at the same time that photographic densities (column 4) of the spectral lines are 
Scientific Papers of the Bureau of Standards, Vol. 16

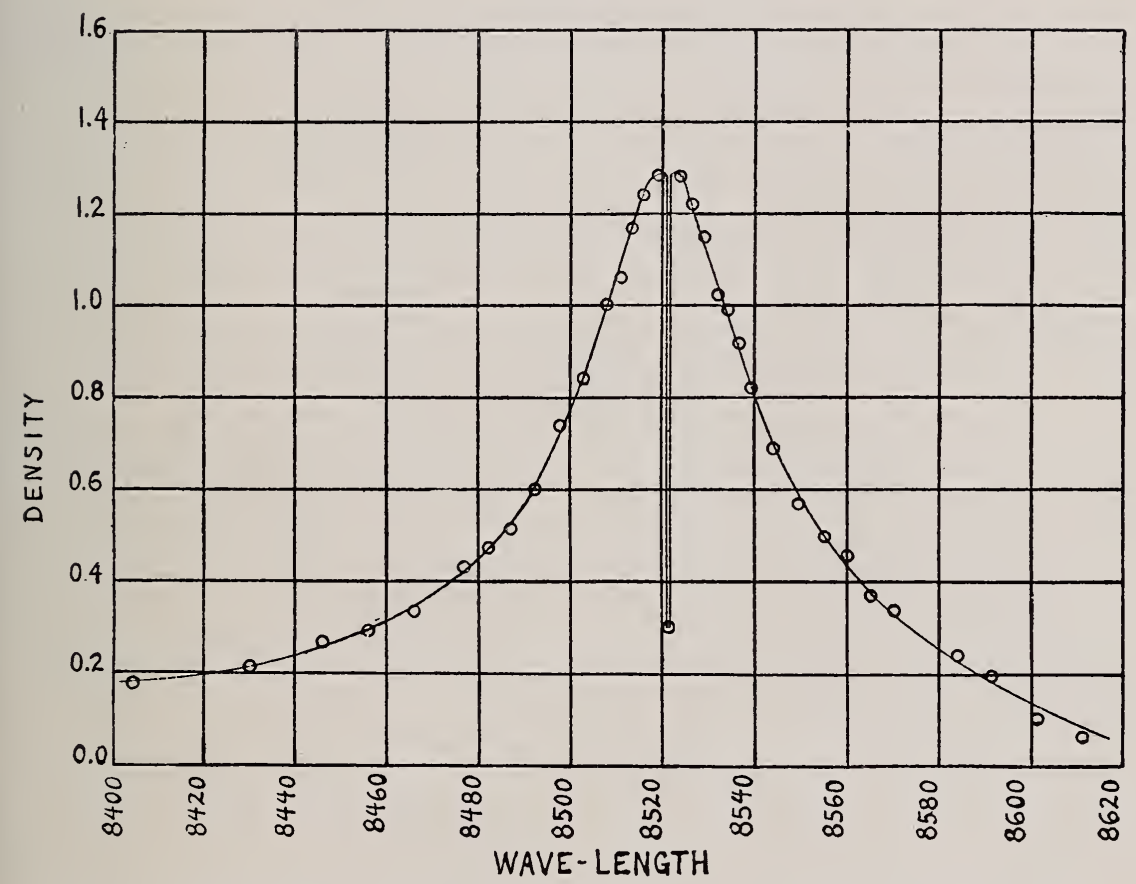

FIG. 3.-Photographic density of the caesium line 8521 A, as shown by a photograph and a plotted curve 
measured. Three wave lengths marked with asterisks were used in wave-length computations by the Hartmann formula.

TABLE 1.-Wave Lengths, Photographic Densities, and Estimated Intensities in Cæsium Arc Spectrum

\begin{tabular}{|c|c|c|c|c|c|}
\hline \multirow{2}{*}{$\begin{array}{l}\text { Micrometer } \\
\text { readings }\end{array}$} & \multicolumn{2}{|c|}{ Wave lengths } & \multirow{2}{*}{$\begin{array}{l}\text { Photographic } \\
\text { densities }\end{array}$} & \multirow{2}{*}{$\begin{array}{l}\text { Estimated } \\
\text { intensities }\end{array}$} & \multirow{2}{*}{$\mathbf{I} / \mathbf{D}$} \\
\hline & Computed & Accepted & & & \\
\hline $\begin{array}{r}178.81 \\
179.47 \\
181.64 \\
2.92 \\
4.01\end{array}$ & $\begin{array}{c}* 4555.30 \\
4592 \\
5656 \\
5837 \\
6006\end{array}$ & $\begin{array}{l}4555.30 \\
4593 \\
5664 \\
5845 \\
6010\end{array}$ & $\begin{array}{r}0.78 \\
.73 \\
.58 \\
1.01 \\
1.08\end{array}$ & $\begin{array}{l}5 \\
5 \\
4 \\
7 \\
8\end{array}$ & $\begin{array}{l}6.4 \\
6.9 \\
6.9 \\
7.0 \\
7.4\end{array}$ \\
\hline $\begin{array}{l}4.15 \\
5.19 \\
5.93 \\
7.04 \\
7.64\end{array}$ & $\begin{array}{l}6029 \\
6211 \\
6353 \\
6586 \\
* 6723.18\end{array}$ & $\begin{array}{l}6034 \\
6213 \\
6355 \\
6587 \\
6723.18\end{array}$ & $\begin{array}{r}.40 \\
1.16 \\
.93 \\
1.04 \\
1.31\end{array}$ & $\begin{array}{l}3 \\
9 \\
7 \\
8 \\
9\end{array}$ & $\begin{array}{l}7.5 \\
7.8 \\
7.5 \\
7.7 \\
6.9\end{array}$ \\
\hline $\begin{array}{r}8.05 \\
8.26 \\
8.67 \\
9.53 \\
199.72\end{array}$ & $\begin{array}{l}6825 \\
6878 \\
6975 \\
7229 \\
7286\end{array}$ & $\begin{array}{l}6827 \\
6873 \\
6973 \\
7229 \\
7280\end{array}$ & $\begin{array}{r}.59 \\
.93 \\
1.38 \\
1.03 \\
.93\end{array}$ & $\begin{array}{r}4 \\
7 \\
10 \\
8 \\
7\end{array}$ & $\begin{array}{l}6.8 \\
7.5 \\
7.2 \\
7.8 \\
7.5\end{array}$ \\
\hline $\begin{array}{r}200.72 \\
1.61 \\
1.80 \\
1.95 \\
2.92 \\
203.73\end{array}$ & $\begin{array}{l}7615 \\
7945 \\
8024 \\
8086 \\
* 8521.12 \\
8946\end{array}$ & $\begin{array}{l}7610 \\
7944 \\
8017 \\
8080 \\
8521.12 \\
8943\end{array}$ & $\begin{array}{r}.76 \\
.45 \\
.26 \\
.17 \\
1.16 \\
.28\end{array}$ & $\begin{array}{l}5 \\
3 \\
2 \\
1 \\
9 \\
2\end{array}$ & $\begin{array}{l}6.6 \\
6.7 \\
7.7 \\
6.0 \\
7.7 \\
7.2\end{array}$ \\
\hline
\end{tabular}

In column 5 the visually estimated intensities of the spectral lines are given. These estimates were made according to the usual practice in spectroscopy of assigning values from I to Io to the intensities of spectral lines recorded on a photographic plate. According to Fechner's law, that "the intensity of sensation increases as the logarithm of the stimulus," the natural tendency in formulating a scale of opacities is to choose a geometric series so that the ratio of the transmissions of any two adjacent steps is constant throughout the series. In other words, the apparent contrasts between successive steps are equal only if the logarithms of the transmissions differ by equal amounts. Visually estimated intensities of spectral lines appear to obey this law very approximately, as is shown in the last column of Table $\mathrm{I}$, in which is given the ratios of visually estimated intensities to measured photographic densities. If the estimated intensities are divided by 7 the quotients will be in close agreement with the photographic densities or logarithms of opacities. ${ }^{6}$

\footnotetext{
${ }^{6}$ It is readily possible, often advisable, and sometimes the practice of spectroscopists to tuse more than Io degrees of opacity in estimating intensities of spectral lines. The average spectrogram perhaps does not give a density of more than 2 for the strongest line on the plate and this suggests that the upper limit of the scale of estimated intensities might conveniently be extended to 20 or even to 200 . Then a larger number of steps would be available in a finer discrimination of opacities, and if the estimates follow Fechner's law, the factor ro or roo will readily permit a rough but of ten sufficiently precise transformation from visually estimated intensities to photographic densities.
} 
Fig. 3 is an illustration of the possible use of the microphotometer in measuring the distribution of energy in a very wide spectrum line. The photograph represents the appearance of the infra-red line $852 \mathrm{I} .12 \mathrm{~A}$ in the $\mathrm{r} 2 \mathrm{O}$-volt arc spectrum of cæsium as photographed on a dicyanin stained plate with a concave grating giving a scale of ro A per millimeter. The curve was obtained

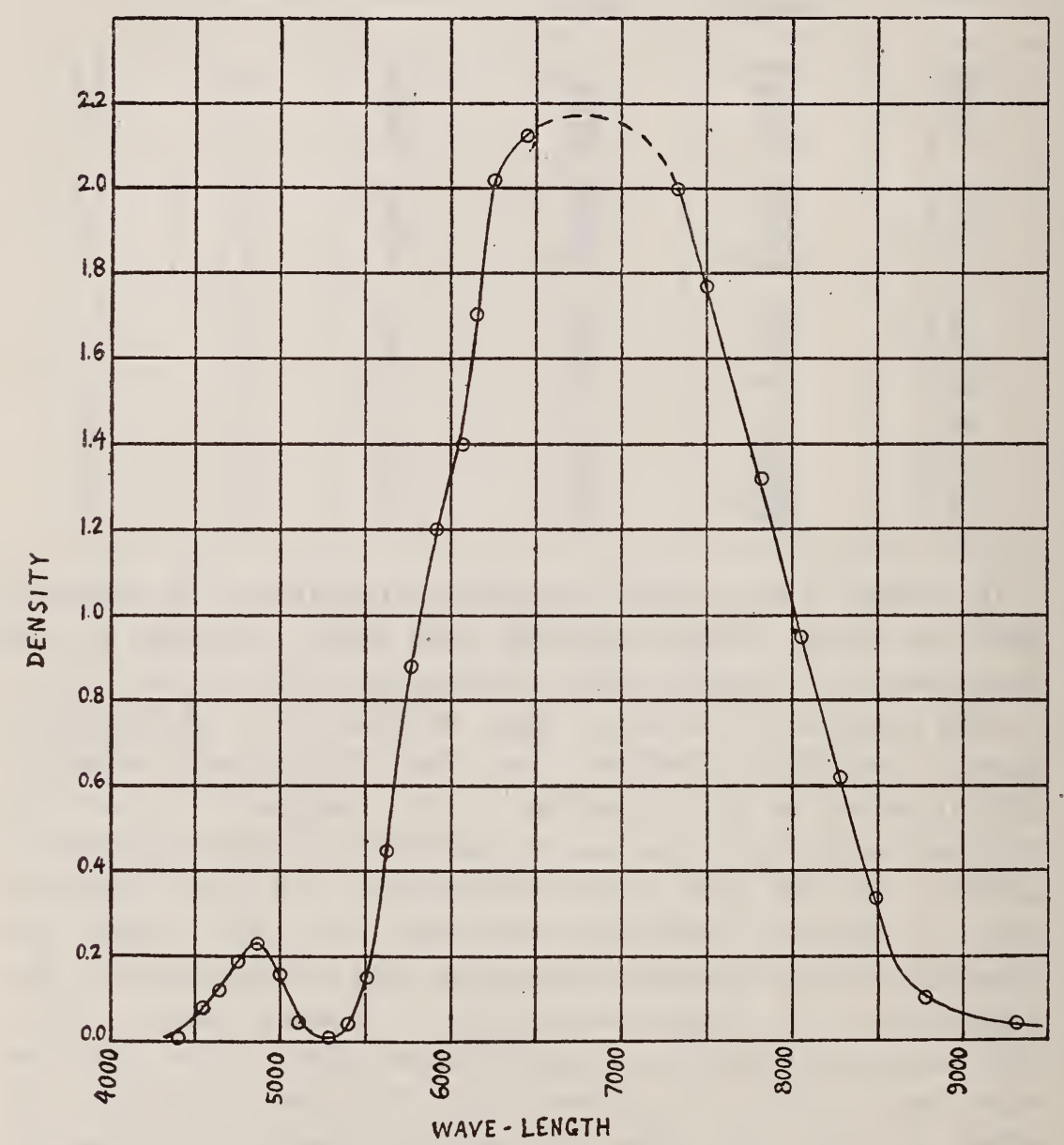

FIG. 4.-Sensitivity of a Seed 23 plate stained with dicyanin

from photographic density measurements with the microphotometer.

This instrument is also well adapted for the measurement of spectral sensitivity of various types of photographic plates after being exposed in a spectrograph to a continuous spectrum. A typical example of this is shown in Fig. 4 which represents microphotometer measurements of density on a Seed 23 plate stained 
with dicyanin and developed after an exposure of one minute in a prism spectrograph which was directed at a black body furnace at $145^{\circ} \mathrm{C}$. The sensitivity of this stained plate to red light appears to be enormously greater than its blue sensitiveness, but the curve should really be corrected to equal energy distribution in the spectrum. To accomplish this, "characteristic curves" for different wave lengths were drawn from microphotometer measurements on exposures of different durations and then the plate sen-

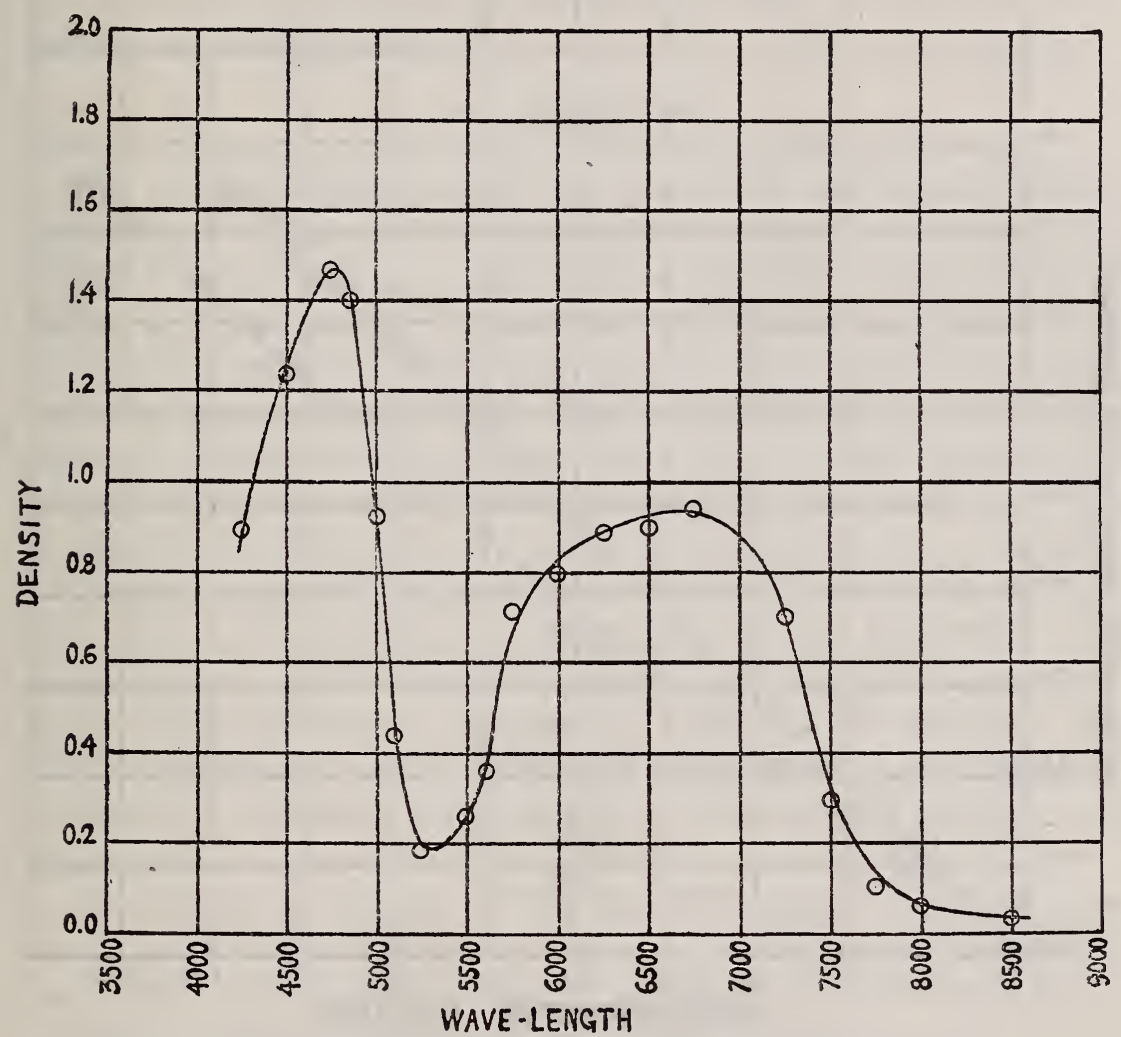

FIG. 5.-Sensitivity of dicyanin stained plate to equal energy spectrum

sitivity was reduced to the relative densities which would be expected if the spectrum possessed equal energy for all wave lengths. This is shown in Fig. 5 .

As a test of the accuracy of density measurements obtained with the microphotometer, some strips of photographic plates prepared in a sensitometer for the purpose of obtaining the $H$ and $D$ characteristic curves ${ }^{7}$ were very carefully measured with a

${ }^{7}$ Hurter and Driffield, Jour. Soc. Chem. Industry, May, I89o, p. 455. 
Koenig-Martens polarization photometer and then with the new microphotometer. The densities measured with the microphotometer were found to be considerably larger than those given by the polarization instrument and the chief reason for this was traced to the manner in which the photographic plate was illuminated in the two instruments. In the polarization photometer the photographic strip is lighted by lamps in a box of opal

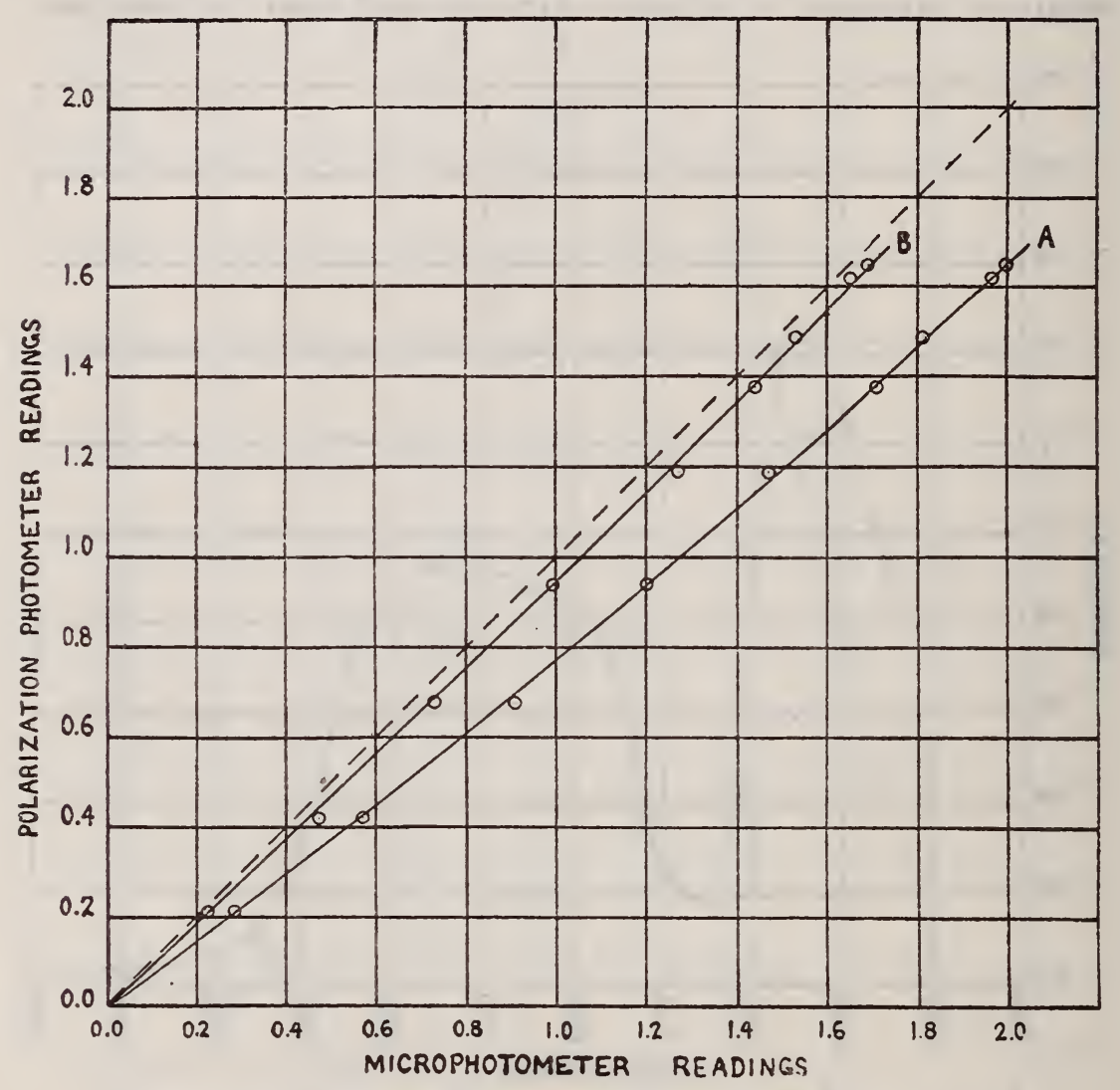

FIG. 6.-Microphotometer $v$. polarization photometer readings

glass so that very diffuse light illuminates the plate. This diffuse light is scattered and partly reradiated by developed silver particles in the photographic plate, and gives the plate apparently a higher transmission and lower density than it really possesses. Practically the same low densities were obtained with the microphotometer when two layers of diffusing ground glass were placed just behind the photographic plate. This is illustrated in Fig. 6, in which the densities of a sensitometer plate as measured with 
the polarization photometer are plotted as ordinates, and those measured with the pyrometer microphotometer as abscissæ. If both instruments gave the same densities the values would lie on the dashed line at $45^{\circ}$. Curve $A$ shows densities determined with the microphotometer when the photographic plate was illuminated with parallel light and curve $B$ when diffuse light from two layers of ground glass illuminated the plate. This experience calls attention to the importance of specifying the character of the illumination when measurements are made on the light transmission of diffusing media. ${ }^{8}$

\section{SUMMARY}

The new microphotometer for measuring photographic densities is essentially the micropyrometer described in the Bulletin of this Bureau (vol. 9, p. 475; I9I3) except that a microscope of higher power is used. The photographic plate is mounted just below the objective of the microscope on a horizontal bed movable with a graduated screw and is illuminated beneath by an intense beam of light from a tungsten ribbon lamp. Light transmitted by a small portion of the photographic plate, the image of which is adjacent to that of the tip of the pyrometer lamp, is matched with equal filament brightness by adjusting the current through the lamp. The ammeter readings are readily translated into measurements of photographic density. If the tip of the pyrometer lamp filament be regarded as a filar in the eyepiece, the relative positions or wave lengths of spectral lines on a plate are measured at the same time that their photographic densities are measured.

An example of the use of this microphotometer for measuring wave lengths and densities of spectral lines is given and it appears that the visually estimated intensities of such lines are a geometric series in accordance with Fechner's law.

Illustrations of this microphotometer's application to the measurement of energy distribution in a broad spectral line and to the measurement of spectral sensitivity of a photographic plate are given.

${ }^{8}$ Absorption and diffusion of light in the developed photographic plate have been studied by Callier, who also presents some of the photographic consequences of the scattering of light. Zeitschr. $\mathrm{f}$. Wiss. Phot., 7, p. 257; rgog. 
Measurements of photographic density when the plate is illuminated by parallel light are found to be much larger than when the illumination is diffuse. This experience calls attention to the importance of specifying the character of the illumination when measurements are made on light transmission of diffusing media.

WASHINGTON, January 20, 1920. 